\title{
Computer control of eye position and velocity
}

\author{
DAVID L. SPARKS and RICHARD HOLLAND \\ Departments of Psychology and Physiology and Biophysics \\ University of Alabama in Birmingham, Birmingham, Aiabama 35294
}

\begin{abstract}
A laboratory computer system is described that permits direct control of eye position and velocity in rhesus monkeys. Using this system, we are able to measure the activity of single neurons while the eye is fixated at specific positions and while the eye is moving, at selected velocities, from one position to another. Illustrative eye movement and spike data are presented, and a brief description of the software used in this system is given.
\end{abstract}

Our research has primarily been concerned with the role of brainstem neurons in the control of visually guided eye movements. To facilitate this work, a chronic microelectrode recording technique and a visual tracking task have been developed for use with the rhesus monkey. The crucial aspect of these techniques for eye movement research is the ability to measure the activity of individual neurons for the fixated eye as well as for the eye moving at constant velocities from one position to another. In the following, a laboratory computer system is described which enables such direct experimental control of the monkey's eye position and velocity along with the measurement of attendant neuronal activity. This system: (a) generates and controls the position and velocity of a visual tracking target presented on an oscilloscope; (b) generates a serial pulse code of target parameters which is recorded on analog tape; (c) monitors horizontal and vertical eye position; (d) compares horizontal and vertical eye position with target position; (e) delivers reinforcement for appropriate tracking of the visual target; (f) provides an on-line display of eye and target position; $(\mathrm{g})$ provides an on-line display of spike activity; and (h) permits modification of target parameters based upon the response properties of each neuron studied.

\section{HARDWARE}

The computer system consists of a PDP-8/I with $12 \mathrm{~K}$ memory. Peripheral equipment includes an AX08 laboratory peripheral device [incorporating two channels of 9-bit digital-to-analog (D/A) conversion, 16 channels of analog-to-digital (A/D) conversion, 12 digital input lines, 12 relay output lines], a Model 1351 Tennecomp cartridge tape unit, a Tektronix 4010A display terminal with hardcopy unit, and two 12-bit D/A channels interfaced to a Hewlett-Packard 1310A oscilloscope (11 x 15-in. CRT viewing area).

This project was supported by National Institutes of Health Grant EY01189. We thank Dr. David Robinson and Dr. Alex Skavenski for their assistance in developing the eye movement recording technique and $\mathrm{Mr}$. Barton Guthrie for technical and programming assistance.

\section{BEHAVIORAL METHODS}

Horizontal and vertical eye movements are measured using a method described previously (Fuchs \& Robinson, 1966). Briefly, a coil of fine wire is surgically implanted beneath the bulbar conjunctiva and recti insertions and the ends of the coil are led to a connector mounted on the monkey's skull. With the head restrained, exposure of the animal to two alternating magnetic fields in spatial and phase quadrature generates signals in the eye coil. These signals are then phase detected to produce two voltages-one proportional to horizontal eye position and one proportional to vertical eye position.

During training and recording sessions, animals are seated in a primate chair with head restrained. The oscilloscope placed $14 \mathrm{in}$. in front of the subject is used to present targets for the visual tracking task. At this distance, maximum horizontal excursion of the target is $\pm 25 \mathrm{deg}$; maximum vertical excursion $\pm 20 \mathrm{deg}$. Two 12-bit D/A converters controlled by the PDP-8/I are used to control the horizontal and vertical positions of the target. The target is a dot subtending a visual angle of less than .l deg and may be presented upon application of a voltage level. Appropriate programs permit this target to be turned on or off at any specified position of a 4096 by 4096 matrix on the screen or to be moved at specified rates from one position to another.

Upon completion of preliminary training and calibration of the eye movement recording system, target presentations and reinforcement are under computer control. During training or data collection trials, horizontal and vertical eye position signals are sampled each $10 \mathrm{msec}$ and compared with target position. "On target" is defined by comparing each sample of horizontal eye position with a horizontal window and vertical eye position with a vertical window. If horizontal eye position exceeds the lower threshold without exceeding the upper boundary of the horizontal window, horizontal eye position is defined as "on target." Similarly, the vertical eye position signal is compared to a vertical window. Window aperture is one 


\section{STANDARD TRACKING TASK}

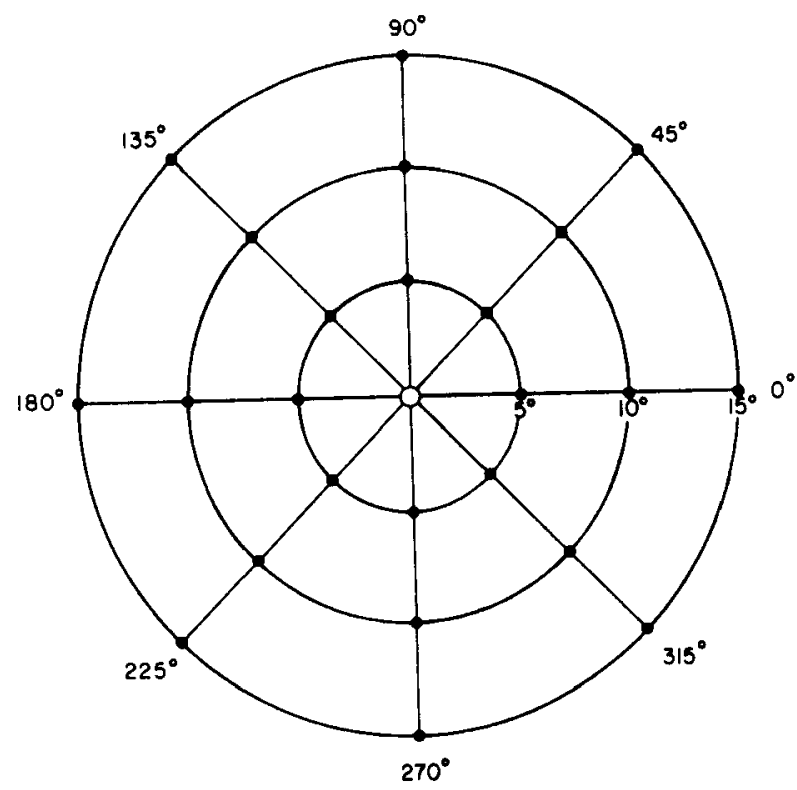

Figure 1. Standard tracking task. If the subject fixates the center dot for $2 \mathrm{sec}$, the target is moved to one of the 24 positions indicated by the filled circles. On saccadic trials, the target movement is instantaneous. On pursuit trials, targets with a 15-deg radius and a fixed velocity of $15 \mathrm{deg} / \mathrm{sec}$ are most commonly used.

of the parameters set by the experimenter during an initial interactive dialogue. Apertures as small as $\pm 1 / 4 \mathrm{deg}$ are used in our experiments although an aperture of \pm 1 deg is most commonly employed.

\section{DATA COLLECTION}

Upon completion of training, the animal is anesthetized, and a stainless steel receptacle for a hydraulic microdrive is secured to the skull. Teflon-coated tungsten microelectrodes may be inserted through this receptacle in order to record, extracellularly, spike potentials from regions of the brainstem involved in the neural control of eye movement.

When a neuron is sufficiently isolated from background noise, the following typical data collection procedure is used: (1) The response of the neuron to spontaneous eye movements and to a standard tracking task is determined. The standard tracking task consists of 24 trials. Each trial starts when the target appears in the center of the screen. The animal is required to fixate the target for $2 \mathrm{sec}$, and the target is then moved instantaneously to one of the 24 positions shown in Figure 1. (2) Each neuron encountered is also tested for its response to slow pursuit movements. Beginning at the center point of the screen, the animals are required to track a target moving at a constant velocity along each of the vectors shown in Figure 1. A velocity of $15 \mathrm{deg} / \mathrm{sec}$ is routinely used, although this parameter may be varied by the experimenter. (3) If the unit responds to eye movements during spontaneous movements or during either tracking task, the movement field of the unit is plotted by having the monkey make saccades and/or smooth pursuit movements to many different positions in the visual field.

During data collection trials, the $\mathrm{x}$ origin, $\mathrm{y}$ origin, target or window size, and angle and radius of each target movement are controlled by the experimenter via on-line interaction with the computer. Additionally, the experimenter receives an on-line display (with optional hard copy) of the spike frequency at the end of each trial.

\section{RESULTS}

We have trained six animals using these procedures. The task is easily learned, and the entire training period does not exceed 3 or 4 weeks.

Figures 2 and 3 illustrate typical horizontal and vertical eye movement records obtained during saccadic and pursuit tracking. The direct control of both eye position and velocity is apparent. Figure 4 illustrates an on-line display of the spike frequency of a brainstem neuron obtained using this system. The spike potential is first converted into a standard pulse using a Schmitt trigger and a monostable multivibrator. An on-line representation of the neuronal spike frequency is then obtained by electronically integrating the standard pulse and sampling the output of the integrator each $25 \mathrm{msec}$.

\section{VISUAL TRACKING SACCADIC MOVEMENTS}

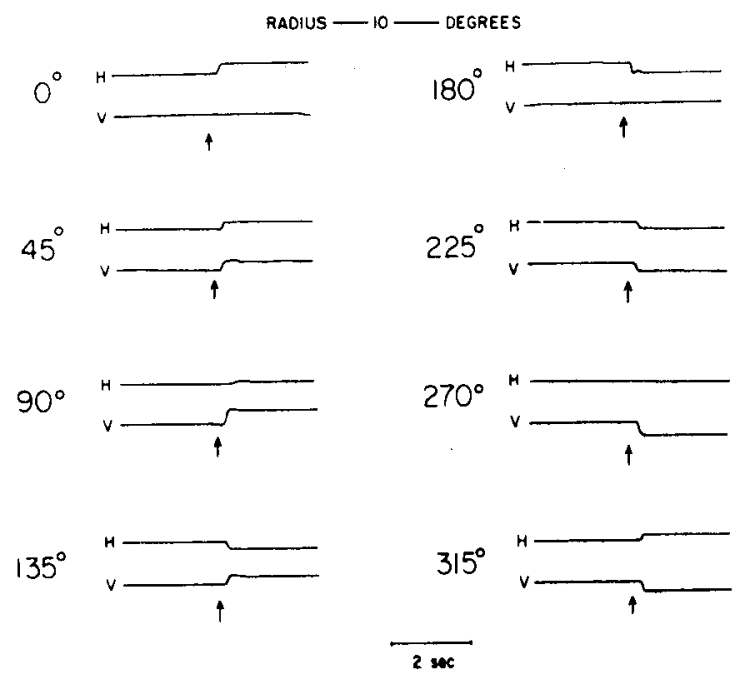

Figure 2. Typical horizontal (H) and vertical (V) eye movements during saccadic trials of the visual tracking task. The target movement (indicated by the arrow) was $10 \mathrm{deg}$ in radius with the angle of movement varied in 45-deg increments from 0 to $15 \mathrm{deg}$ (see Figure 1). An upward deflection of the tracing indicates right and up movements for the horizontal and vertical channels, respectively. 
VISUAL TRACKING-PURSUIT MOVEMENTS
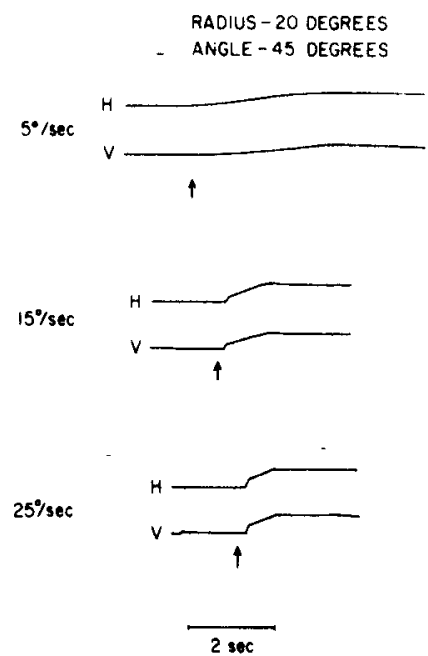

Figure 3. Typical horizontal (H) and vertical (V) eye movements during pursuit tracking. The target movement (onset indicated by the arrow) traversed a $15-\mathrm{deg}$ radius at an angle of $45 \mathrm{deg}$, and at the three velocities indicated. Note that after a saccade to acquire the sudden onset of target movement, the velocity of both horizontal and vertical movements matches target velocity.

\section{SOFTWARE}

Programs for these experiments utilize an interaction of FOCAL and assembly language subroutines similar to that described for the PDP-12 (Siegel, 1972). FOCAL is used for specifying parameters, binary-to-decimal and decimal-to-binary conversions, and mathematical routines. Assembly language is used for stimulus display, timing, A/D conversions, and other components of actual data collection where timing is critical. In our $12 \mathrm{~K}$ machine, Field 0 is reserved for assembly language programs, interrupt service routines, and Tektronix 4010 display terminal routines. Field $\mathbf{I}$ is reserved for data and variable storage. Field 2 contains a modified version of 4K FOCAL which permits interaction with Memory Fields 0 and 1 .

The interaction of FOCAL and assembly language subroutines is achieved by calling a single FNEW function which utilizes features of FOCAL overlays previously described (Johnson, 1973; Reece, 1973). However, the first argument of this function is an index to a table and may actually specify one of eight functions. Examples and brief descriptions of these functions are given below.

\section{SET Z $=$ FNEW $($ JMS,$A)$}

Execution of this line transfers control to Decimal Location $\mathrm{A}$ in Field 0 which is the initial address of an assembly language subroutine. Upon completion of the subroutine in Field 0, FOCAL is reentered at the next line or instruction.

\section{SET Z $=$ FNEW $($ GET,$A)$}

Execution of this line gets the contents of Decimal Location A in Field 1. FOCAL interprets the 12-bit binary value as a decimal number in the range 04096 . The GET instruction may be used to obtain a variable string from Field 1 core. The following instruction illustrates this point: FOR I=6,10; SET Z(I)=FNEW $($ GET, I $)$.

\section{SET Z $=$ FNEW $($ PUT $, A, B)$}

This instruction transfers an integer from FOCAL (Field 2) into Field 0. The parameter $A$ is the destination address and $B$ is the integer to be moved. $A$ and $\mathrm{B}$ must be the decimal equivalents of the desired octal values.

\section{SET $Z=F N E W(I N L, X, Y)$}

This is a command to set the cursor of the Tektronix 4010 display to a given $x, y$ point without intensifying the beam.

\section{SET Z=FNEW(PNT $, X, Y)$}

This is a command to plot a point at $\mathrm{x}, \mathrm{y}$ on the 4010 terminal.

\section{SET Z $=$ FNEW $(V E C, X, Y)$}

This is a command to plot a vector from the current position of the cursor to $x, y$.

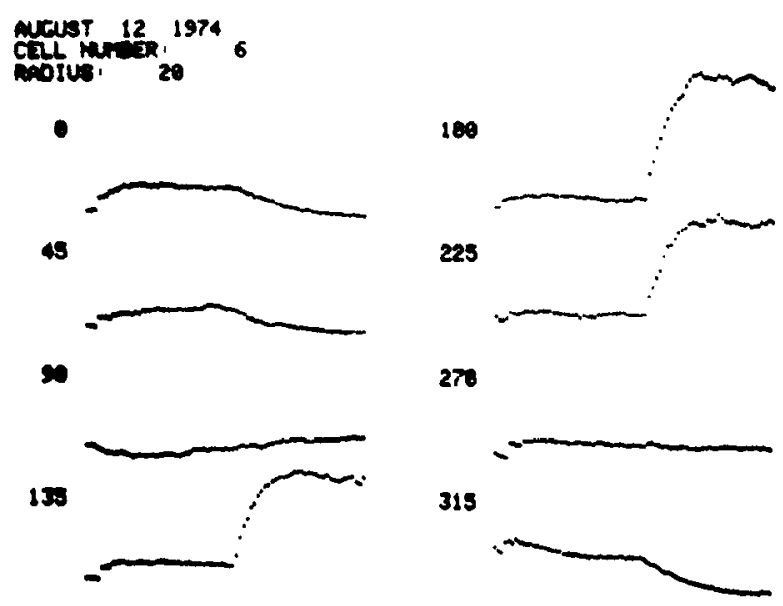

Figure 4. An on-line display of relative spike frequency of a brainstem neuron recorded during eight visual tracking trials. Each trace represents spike frequency occurring during $4 \mathrm{sec}-2 \mathrm{sec}$ at center position and $2 \mathrm{sec}$ following movement of the target to a second position. The target movement was $20 \mathrm{deg}$ in radius and the angle of the movement is shown to the left of each trace. Note that the spike frequency of this neuron increases during left fixations, decreases during right fixations, and is unaltered during fixations along angles of $90 \mathrm{deg}$ and 270 deg. 


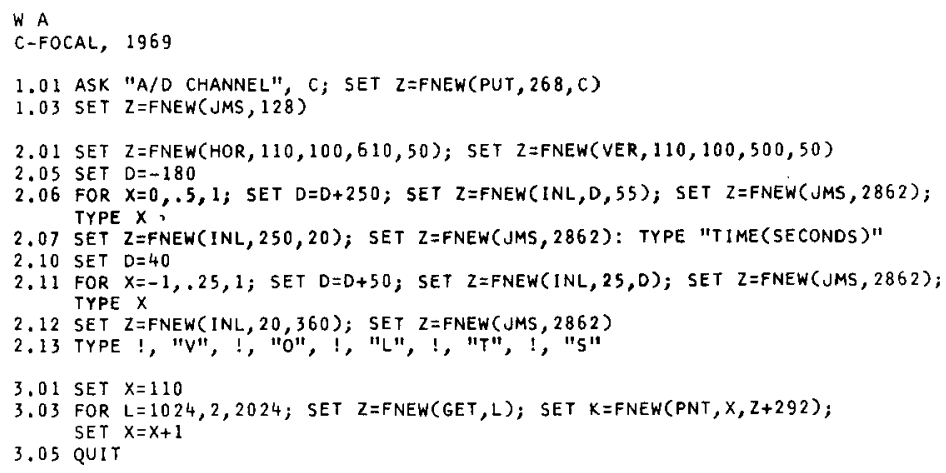

\section{SET Z=FNEW(VER,A,B,C,D)}

This is a command to draw a vertical axis where $A$ is the $\mathrm{x}$ origin, $\mathrm{B}$ is the $\mathrm{y}$ origin, $\mathrm{C}$ is the end point of the axis, and $D$ is the number of spaces between reference marks on the axis.

\section{SET $Z=F N E W(H O R, A, B, C, D)$}

This is a command to draw a horizontal axis where $\mathrm{A}$ is the $\mathrm{x}$ origin, $\mathrm{B}$ is the $\mathrm{y}$ origin, $\mathrm{C}$ is the end point of the axis, and $D$ is the number of spaces between reference marks on the axis.

The program shown above was constructed to illustrate the use of these functions. The output of the program is shown in Figure 5. Line 1.01 asks which A/D channel is to be sampled and uses the PUT function to deposit this value as an operand in Field 0, the field containing the assembly language routine. Line 1.03 transfers control to Field 0 via the JMS function. The assembly language routine samples the selected A/D channel once each millisecond for a $1-\sec$ period and stores these values in Field 1, the field reserved for variables and data. At the end of $1 \mathrm{sec}$, FOCAL is reentered at Line 2.01 .

Line 2.01 draws the horizontal and vertical axes. Lines 2.05, 2.06, and 2.07 label the abscissa and Lines 2.10 through 2.13 are used to label the ordinate. It should be noted that before the axes can be labeled, the JMS function is used to execute a subroutine located at $2862_{10}$ in Field 0 which places the 4010 terminal in an alphanumeric mode. A similar command is not required to switch from alphanumeric to graphic mode.

Lines 3.01 and 3.03 plot 500 of the 1,000 points that were stored in Field 1 by the assembly language subroutine. The GET function is used to retrieve the data from Field 1, and the PNT function is used to plot these points.

Several features of this sample program should be noted: (1) The initial section of the program (Line 1.01) specifies the parameters to be used. These parameters may be stored in another memory field as operands or may be used in a subsequent section of the FOCAL program for computation of stimulus or response parameters. (2) The second section of the program (Line 1.03) transfers control to an assembly language subroutine for actual stimulus presentation, data collection, and data storage using the parameters specified in the first section. (3) Upon completion of data collection, FOCAL is reentered and used for data analysis and for printouts or display of the data.

The program for control of eye position and velocity uses an interaction of FOCAL and assembly language in the manner described below. The first section of the program is written in FOCAL and specifies the parameters for the first block of trials. Window size, radius and angle ranges, radius and angle increments, and target velocity are specified. Additional information
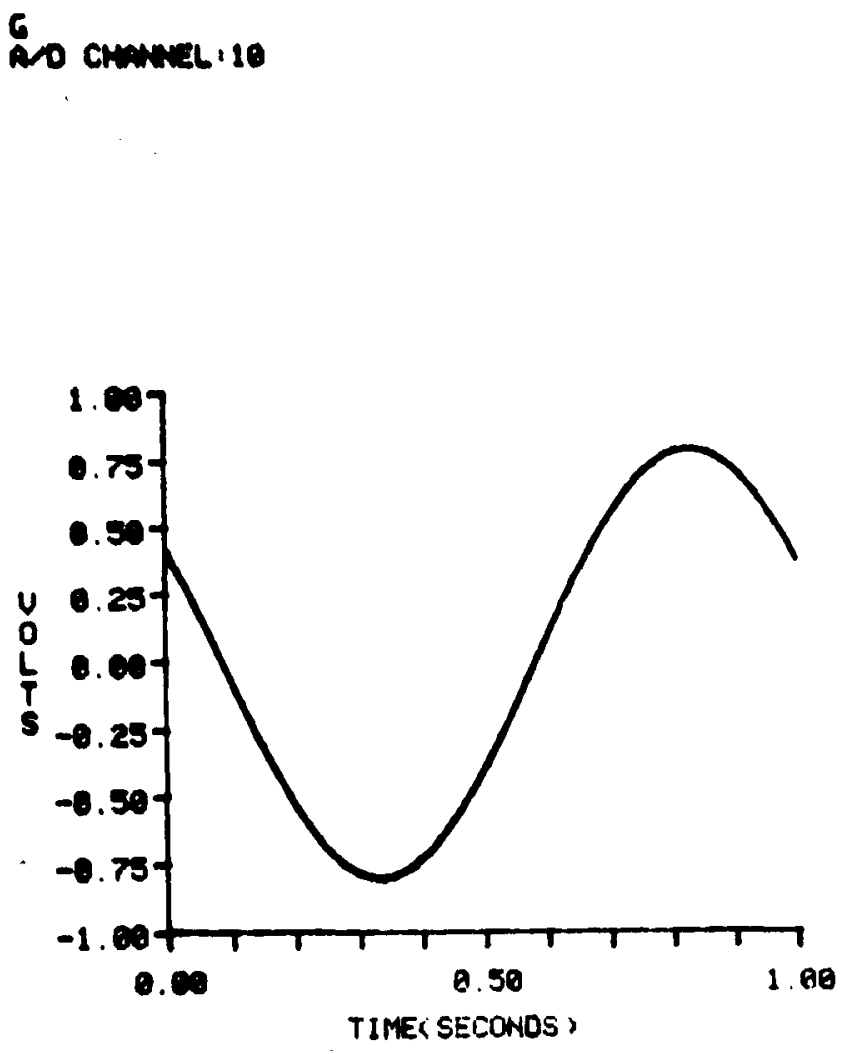

Figure 5. Photograpn of the hard-copy output of a display on the Tektronix $4010 \mathrm{~A}$ terminal. The display was generated by the sample program described in the text. 
concerning date, cell number, subject number, and trial number are requested for use in generating the analog tape heading and for labeling of the on-line data displays.

The second section of the program controls target presentation, the monitoring and comparing of eye and target position and the sampling and storing of spike frequency information. This section of the program is an assembly language subroutine of FOCAL.

Between trials, FOCAL is reentered, the spike data from the previous trial displayed, and the $\mathrm{x}, \mathrm{y}$ parameters of the next target position are computed using appropriate trigonometric functions. These parameters are deposited in the assembly language program and then control is returned to the assembly language subroutine for presentation of the next trial.
From a programming point of view, two major advantages are realized by using a combination of FOCAL and assembly language: (a) the tedious assembly language programming of input-output, binary-to-decimal, decimal-to-binary, and computation of mathematical functions is avoided; and (b) variable storage in FOCAL is increased significantly through use of the PUT function.

\section{REFERENCES}

Fuchs, A. F., \& Robinson, D. A. A method for measuring horizontal and vertical eye movement chronically in the monkey. Journal of Applied Physiology, 1966, 21, 1068-1070.

Johnson, F. R. Letter to the Editor. Decuscope, 1973, 12, 31 .

Reece, $P$. Some simple I/O patches for $4 \mathrm{~K}$ Focal. Decuscope. $1973,12,23-29$.

Siegel, W. Combining FOCAL and Assembly language. Behavior Research Methods \& Instrumentation, 1972, 4, 105-106. 